\title{
Study on multi-ELVES in the Pierre Auger Observatory
}

\section{A. Vásquez-Ramírez ${ }^{a, *}$ on behalf of the Pierre Auger ${ }^{b}$ Collaboration}

(a complete list of authors can be found at the end of the proceedings)

${ }^{a}$ Universidad Industrial de Santander, Escuela de Física, Carrera 27 Calle 9, Bucaramanga, Colombia

${ }^{b}$ Observatorio Pierre Auger, Av. San Martín Norte 304, 5613 Malargüe, Argentina

E-mail: spokespersons@auger.org

Since 2013, the four sites of the Fluorescence Detector (FD) of the Pierre Auger Observatory record ELVES with a dedicated trigger. These UV light emissions are correlated to distant lightning strikes. The length of recorded traces has been increased from $100 \mu \mathrm{s}$ (2013), to $300 \mu \mathrm{s}$ (2014-16), to $900 \mu \mathrm{s}$ (2017-present), to progressively extend the observation of the light emission towards the vertical of the causative lightning and beyond. A large fraction of the observed events shows double ELVES within the time window, and, in some cases, even more complex structures are observed. The nature of the multi-ELVES is not completely understood but may be related to the different types of lightning in which they are originated. For example, it is known that Narrow Bipolar Events can produce double ELVES, and Energetic In-cloud Pulses, occurring between the main negative and upper positive charge layer of clouds, can induce double and even quadruple ELVES in the ionosphere. This report shows the seasonal and daily dependence of the time gap, amplitude ratio, and correlation between the pulse widths of the peaks in a sample of 1000+ multi-ELVES events recorded during the period 2014-20. The events have been compared with data from other satellite and ground-based sensing devices to study the correlation of their properties with lightning observables such as altitude and polarity.

$37^{\text {th }}$ International Cosmic Ray Conference (ICRC 2021)

July 12 th - 23rd, 2021

Online - Berlin, Germany

\footnotetext{
*Presenter
} 


\section{Introduction}

ELVES (Emission of Light and Very low-frequency perturbations due to Electromagnetic pulse Sources) are a class of Transient Luminous Events (TLEs), which occur at the base of the ionosphere above intense electrical storms. The light, emitted in the form of a rapidly expanding ring, is generated by the electromagnetic pulse (EMP) of extreme lightning that propagates as a spherical wave from the base of the lightning channel. The EMP intersects the lower ionosphere and transfers energy to free electrons exciting transitions when they collide with atmospheric molecules. Those energetic molecules return to their lower-energy state, emitting a wide-frequency spectrum of light in a process known as fluorescence [1].

The first observations of ELVES were diffuse airglow in the sky [2], but thanks to the improved sensitivity of the detectors it was possible to measure the rapid lateral expansion of their luminosity [3], and even its photometric traces began to show multiple-peak structures [4-6]. The study of these phenomena has significantly advanced in the last decade with TLE detectors from Space [7$10]$ and from ground $[6,11,12])$; however, there are still open questions about the phenomenology of ELVES.

On the other hand, the Fluorescence Detector (FD) of the Pierre Auger Observatory [13], located in the Mendoza province of Argentina, has been detecting ELVES since 2005 [14]. Its observation footprint for ELVES is $3 \times 10^{6} \mathrm{~km}^{2}$, including the Córdoba region where large thunderstorms occur [6]. The Auger FD technology with a 100 ns time resolution has been used to record the internal structure of ELVES with unprecedented precision. Indeed, the Auger FD has reported on the first ELVES with three peaks in its photo-traces [6]. From now on, we will refer to events with more than one peak in their photo-traces as multi-ELVES. In this report, we show the seasonal distribution of ELVES detected during the period 2014-20. We also present the analysis of a frequently detected type of multi-ELVES, whose temporal difference between its two peaks is approximately constant.

\section{Sources that may produce multi-ELVES}

Currently, it is believed that two-peaks ELVES are originated by intracloud (IC) lightning stroke. The wide time separation between the peaks was observationally confirmed to correlate with high altitude compact intracloud lightning discharges (CIDs) [15]. If an IC lightning occurs at an altitude $H_{b}$, the first peak in the ELVES photo-trace is created by the EMP direct path to the ionosphere, while the second one is created by the ground reflection of the EMP reaching the ionosphere with a time delay [15]. Then, with the time difference $(\Delta T)$ between two peaks, we can reconstruct the bolt altitude $H_{b}$ (see the method in [16]).

In the top panel of figure 1 a multi-ELVES event that could have been produced by this mechanism is shown. The $\Delta T$ vs distance from the lightning stroke data should follow the relationship curves (empty circles curves) for some particular altitude. In this figure, we observe that the data follow the curve corresponding to $H_{b}=20 \mathrm{~km}$ in two FD sites where this ELVES was observed: i.e Los Morados and Coihueco. Two bays observing approximately the same $\Delta T$ values in the zones of the ionosphere where their field of view intersect, is instead shown in the right panel of the same figure. 

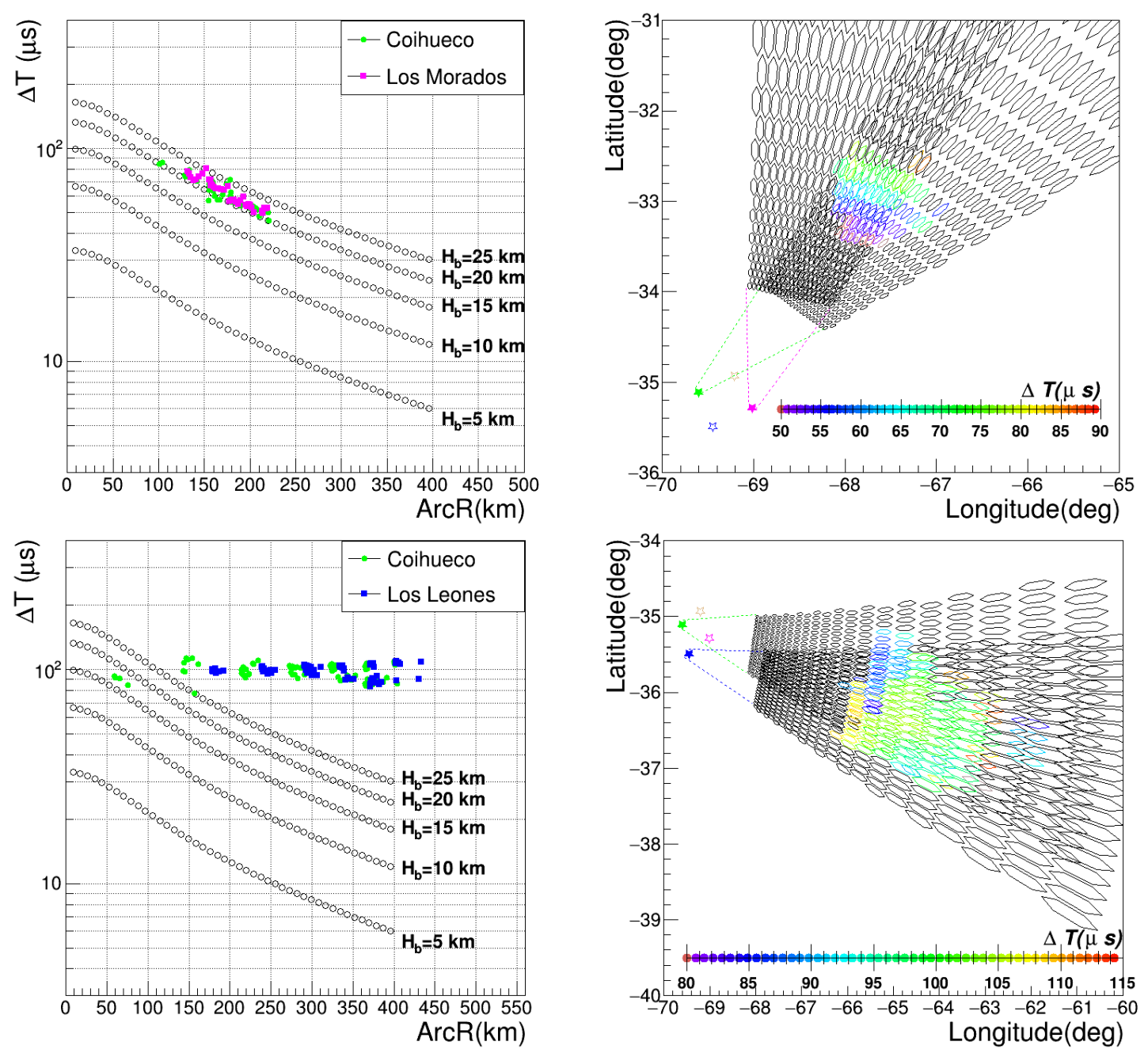

Figure 1: Top panel: multi-ELVES event on the night of February 09, 2018, possibly produced through the EMP ground reflection mechanism. The left panel shows the time difference between peaks from two FD sites (Coihueco and Los Morados) corresponding to a lightning altitude of about $20 \mathrm{~km}$. In the right panel two dimensional surface light emission map of the multi-ELVES, showing in color approximately the same $\Delta T$ values from two independent sites. Bottom panel: multi-ELVES event detected on the night of April 28, 2020, that shows an almost constant $\Delta T$ from two independent FD sites: Coihueco and Los Leones (left panel). In the right panel two dimensional surface light emission map of the multi-ELVES, showing in color the time difference from the two peaks. The same constant time difference is observed from the two independent sites.

However, in the multi-ELVES data, we note that very few events follow this production mechanism. For example, between 2018 and 2020 there are only about 16 ground reflection mechanism candidates, while a large number of events shows an approximately constant $\Delta T$ (see bottom panel of figure 1) and others that do not even follow some functional between $\Delta T$ and distance to the lightning source. In section 3 we show the seasonal distribution of these events to better analyze their frequency of production during years.

Different EMPs sources may produce a diversity of ELVES that depends on the properties of 


\begin{tabular}{|c|c|c|c|c|}
\hline Trace length $(\mu \mathrm{s})$ & Year & Total events & Total ME & Ratio (ME/Total) \\
\hline 300 & 2014 & 508 & 115 & 0.226 \\
300 & 2015 & 673 & 201 & 0.299 \\
300 & 2016 & 695 & 190 & 0.273 \\
\hline 900 & 2017 & 1140 & 323 & 0.283 \\
900 & 2018 & 875 & 233 & 0.266 \\
900 & 2019 & 1689 & 500 & 0.296 \\
900 & 2020 & 1157 & 311 & 0.269 \\
\hline
\end{tabular}

Table 1: The total number of events and the total number of multi-ELVES detected by the FD in the period 2014-20. The number of events increases from 2017 onwards but the ratio of multi-ELVES to the total remains more or less constant.
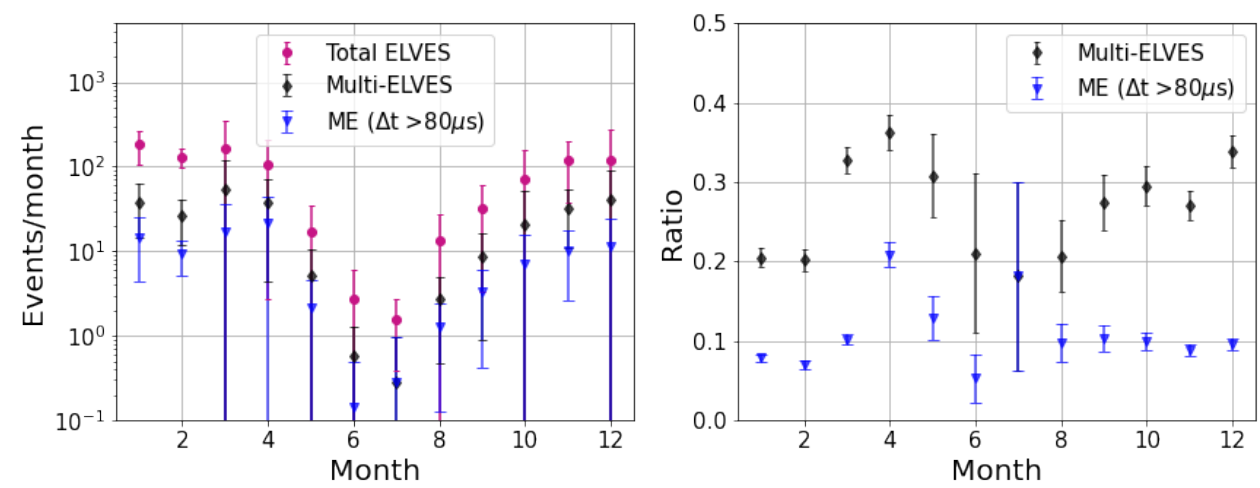

Figure 2: Left panel: monthly distribution of total events, multi-ELVES, and multi-ELVES with $\Delta T>80 \mu \mathrm{s}$ during the period 2014-20. Right panel: monthly ratio of multi-ELVES and multi-ELVES with $\Delta T>80 \mu \mathrm{s}$ to total number of ELVES.

the lightning, such as the type of source, i.e cloud-to-ground (CG), energetic in-cloud pulses (EIPs) or CIDs, its altitude, waveform and duration. For example, ELVES doublets induced by EIPs are less separated in space and time than ELVES induced by CIDs [18]. We do not fully understand how these multi-ELVES are produced but we can analyze their photo-traces, as well as the characteristics of the lightning, to obtain some clues.

The study on multi-ELVES could be useful for understanding other phenomena that occur in storms, called Terrestrial Gamma-ray Flashes (TGFs). Recently it has been found that EIPs associated with some TGFs can produce a current moment waveform with a peak of hundred of $\mathrm{kA} \mathrm{km}$ and a duration of $10 \mu \mathrm{s}$ [18]. These short EIPs are more likely to have accompanying multi-ELVES [18] because their source currents vary more rapidly. Nevertheless, in this report we focus on the analysis of multi-ELVES with constant $\Delta T$, further studies will be necessary to understand the relationship of multi-ELVES with TGFs. 


\section{Seasonal distribution of multi-ELVES}

From 2017 onwards the number of detected events increases compared to the period 2014-16. This is due to the improved trigger implemented in 2017 [5]. In table 1 it is summarised the total number of events, the total number of multi-ELVES, and the ratio for the period 2014-20. With the extension of the readout system, from $300 \mu \mathrm{s}$ to $900 \mu$ s trace length, we could expect an increase in the number of detected multi-ELVES to the total number of events since a longer trace allows to observe events with two peaks far apart from each other. However, the annual ratio of multi-ELVES to total events remains more or less constant, and over the period 2014-20 it is around 0.273 \pm 0.023 .

The seasonal distribution of multi-ELVES can be studied by plotting the mean number of ELVES detected per month in the FD. This distribution is shown in figure 2 for the total events, multi-ELVES and multi-ELVES with $\Delta T>80 \mu$ s in the period 2014-20. The highest frequencies of events occur around the southern summer and autumn.

On the other hand, the monthly ratio of multi-ELVES to total is shown in the right panel of figure 2, where we observe that the ratio of multi-ELVES to total is higher during early summer (December) and autumn (March-May), while for multi-ELVES with $\Delta T>80 \mu$ s the ratio is higher in April.

\section{Analysis of multi-ELVES with constant time difference}

The night of April 28, 2020, has been selected to have the highest number of events with $\Delta T>80 \mu$ s during the period 2014-20. In this night a total of 144 ELVES were detected, of which 1 could be a multi-ELVES ground reflection mechanism candidate and 33 are multi-ELVES with almost constant $\Delta T>80 \mu \mathrm{s}$.

For a finer analysis of the multi-ELVES photo-traces, we calculate the ratio between the total quantity of light from the first peak $\left(Q_{1 \text { tot }}\right)$ respect to the total quantity of light from the second peak $\left(Q_{2 \text { tot }}\right)$ of each pixel signal. In figure 3 we show the ratio profile $Q_{2 \text { tot }} / Q_{1 \text { tot }}$ vs the distance to the lightning bolt of the two multi-ELVES reported in section 1. We notice that the multi-ELVES with almost constant $\Delta T>80 \mu \mathrm{s}$ (bottom panel) shows a different profile from the EMP ground reflection event (top panel). This profile has the same structure from the two observation sites of the FD (Coihueco and Los Morados). In the right panel of the same figure, we observe that both FD sites detect similar $Q_{2 \text { tot }} / Q_{1 \text { tot }}$ profile in the zones of the ionosphere where their fields of view overlap.

In order to complement the analysis of these multi-ELVES with the characteristics of the lightning that produced them, we correlated the events from this night with the data provided by the Earth Networks Total Lightning Network (ENTLN) [19] and the World Wide Lightning Location Network (WWLLN) [20]. Out of a total of 144 ELVES, 128 were correlated. From the correlation we obtain the location in latitude and longitude as well as the peak current of the lightning strike. In figure 5 we show the location of lightning inducing the total ELVES of the night (violet stars), and those that generated multi-ELVES with almost constant $\Delta T>80 \mu$ s (blue stars).

From previous correlation of Auger events with GLD360 data set during the 05/2017-12/2018 period, we observed an asymmetric polarity distribution in the peak current of lightning inducing single, double and multi-ELVES. Figure 4 shows that single ELVES are positive polarity dominated 

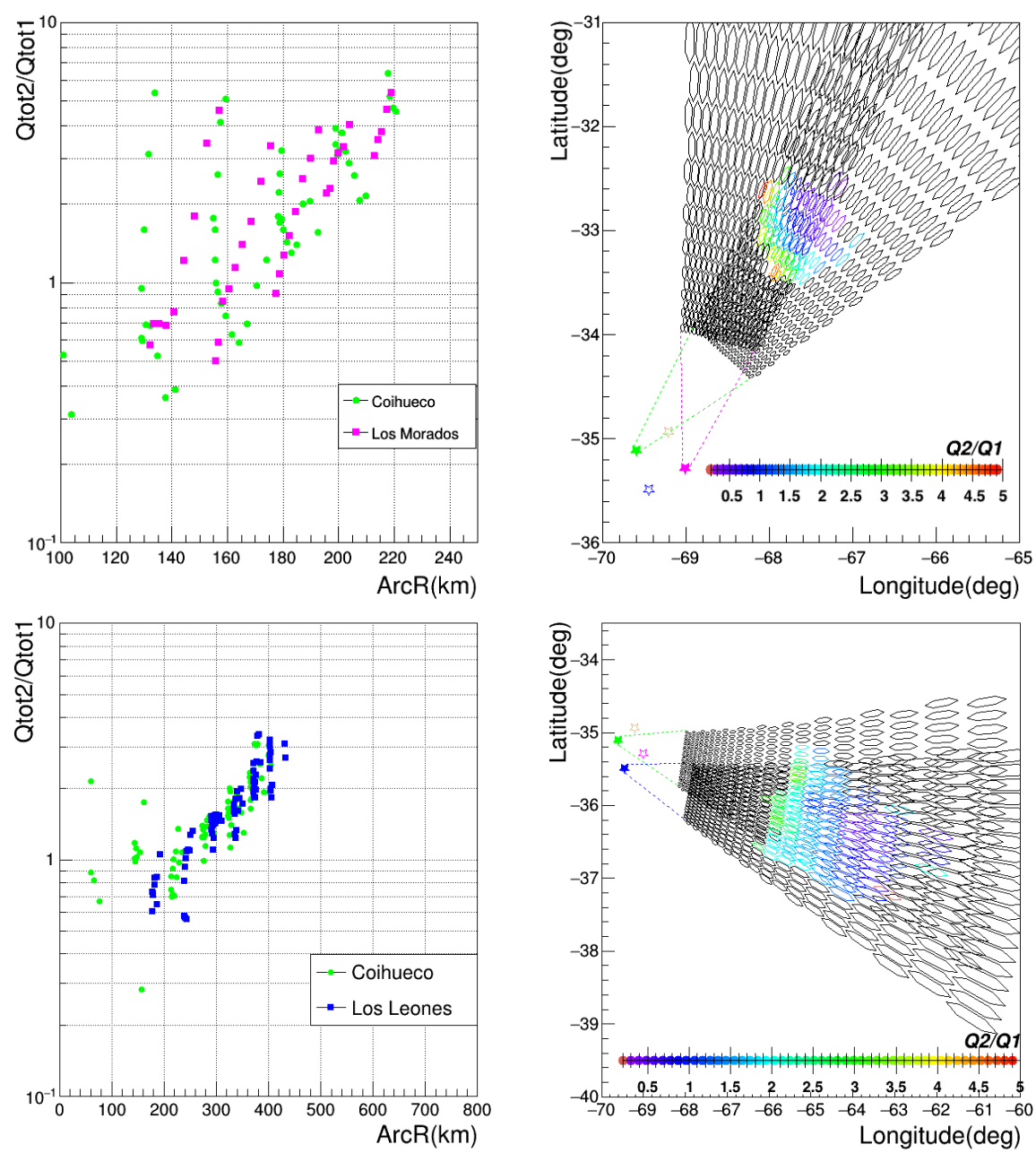

Figure 3: Left panel: ratio profile of the total light from the second peak $\left(Q_{2 \text { tot }}\right)$ respect to the first peak ( $Q_{1 \text { tot }}$ ) of the multi-ELVES traces vs the distance to the lightning bolt of two multi-ELVES reported in figure 1. In the right panel two dimensional surface light emission map of the multi-ELVES, showing in color the $Q_{2 \text { tot }} / Q_{1 \text { tot }}$ ratio from two independent sites. The multi-ELVES with almost constant $\Delta T>80 \mu$ s (bottom panel) shows a different $Q_{2 \text { tot }} / Q_{1 \text { tot }}$ pattern from the $Q_{2 \text { tot }} / Q_{1 \text { tot }}$ EMP ground reflection event profile (top panel).

while double and multi-ELVES are negative polarity dominated. We perform a similar analysis with ELVES of April 28, 2020. Right panel of figure 5 shows that most of the lightning that produced multi-ELVES with $\Delta T>80 \mu$ s had a negative polarity as did most of the lightning that produced the total of ELVES, i.e. this thunderstorm shows a different polarity distribution than expected from the correlated events in the figure 4 . Therefore, it is necessary to analyze the polarity distribution of each type of storm where there is a high frequency of multi-ELVES.

\section{Final remarks}

The technical capabilities of the Auger FD, especially its high temporal resolution of $200 \mathrm{~ns}$, allow the study of multi-ELVES traces in great detail. During the period 2014-20, there are very 


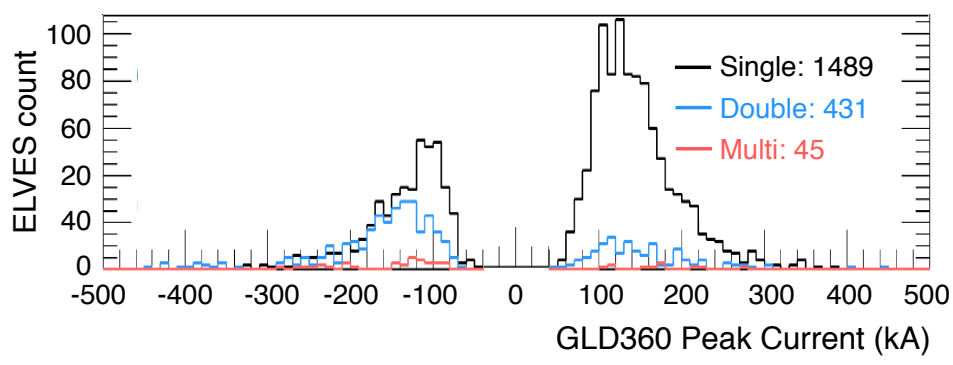

Figure 4: The GLD360 peak current (and polarity) of the sources of EMPs that induced single-, double-, and multi-peaked ELVES, in coincidence with Auger data during the 05/2017-12/2018 observation period.
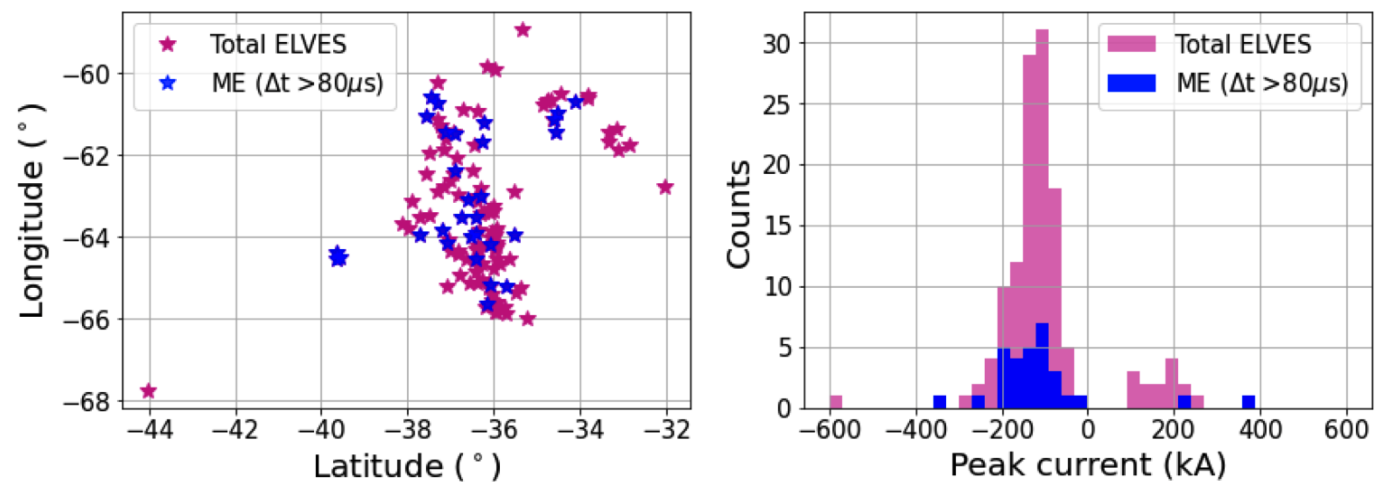

Figure 5: Events correlated with ENTLN and WWLLN data. Left panel: the location of lightning inducing the total of ELVES (violet stars) and those that produced multi-ELVES with almost constant $\Delta T$ (blue stars). Right panel: the peak current distribution of the lightning.

few candidates to be explained by the EMP ground reflection mechanism while a very frequent type of multi-ELVES shows an almost constant difference between the peaks and greater than $80 \mu \mathrm{s}$.

From 2017 onwards the number of events increases compared to the period 2014-2016, however, the annual ratio of multi-ELVES to total events remains rather constant (around $0.273 \pm 0.023$ ). The seasonal distribution of ELVES detected during the period 2014-20 shows that highest frequencies of events occur around the southern summer and autumn. On the other hand, the ratio of multiELVES to total is higher during early summer and autumn, while for multi-ELVES (ME) with $\Delta T>80 \mu$ s the ratio is higher in April.

Finally, we show the analysis of the events detected at the night with more multi-ELVES of constant $\Delta T$ (April 28, 2020). The ratio profile $Q_{2 \text { tot }} / Q_{1 \text { tot }}$ of a multi-ELVES with constant $\Delta T$ is different from the profile of a EMP ground reflection mechanism candidate.

In the other hand, out of 144 ELVES 128 were correlated with ENTLN and WWLLN data. The peak current distribution of this thunderstorm differs from the distribution obtained from the correlation of Auger data with the GLD360, during the 05/2017-12/2018 observation period. Therefore, it is important to analyze the peak current distribution for each type of storm, as well as other characteristics of lightning, such as the type of lightning or the waveform of the electric field variation signal. 


\section{References}

[1] Y. N. Taranenko et al., Geophys. Res. Lett. 20 (1993) 2675-2678.

[2] W. L. Boeck et al., Geophys. Res. Lett. 19 (1992) 99-102.

[3] U. S. Inan et al., Geophys. Res. Lett. 24 (1997) 583-586.

[4] R. T. Newsome and U. S. Inan, J. Geophys. Res.-Space 115 (2010) .

[5] R. Mussa [for the Pierre Auger Coll.], EPJ Web Conf. 197 (2019).

[6] A. Aab et al. [Pierre Auger Coll.], Astr. Soc. P. 7 (2020).

[7] C. L. Kuo et al., J. Geophys. Res.-Space 117 (2012) .

[8] M. Sato et al., IEEJ Trans. Fundam. Mater. 131 (2011) 994-999.

[9] T. Ushio et al., IEEJ Trans. Fundam. Mater. 131 (2011) 971-976.

[10] T. Neubert et al., Space Sci. Rev. 215 (2019) 1-17.

[11] O. A. van der Velde and J. Montanyà, Geophys. Res. Lett. 43 (2016) 5467-5474.

[12] R. Marshall et al., IEEE Trans. Geosci. Remote Sens. 46 (2008) 3885-3893.

[13] J. Abraham et al. [Pierre Auger Coll.], Nucl. Instrum. Meth. A 620 (2010) 227-251.

[14] R. Mussa [for the Pierre Auger Coll.] and G. Ciaccio, Eur. Phys. J. Plus 127 (2012) 1-6.

[15] R. A. Marshall et al., Geophys. Res. Lett. 42 (2015) 6112-6119.

[16] R. Mussa [for the Pierre Auger Coll.], Proc. 36th Int. Cosmic Ray Conf., Madison, Wisconsin, USA (2019), PoS(ICRC2019)1131, [1909.09073].

[17] T. Neubert et al., Science 367 (2020) 183-186.

[18] N. Liu et al., J. Geophys. Res. 122 (2017) 10-563.

[19] https://www. earthnetworks.com/.

[20] http://wwlln.net/. 


\section{The Pierre Auger Collaboration}

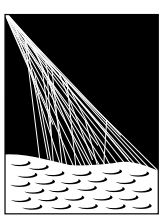

PIERRE

AUSGERVATORY

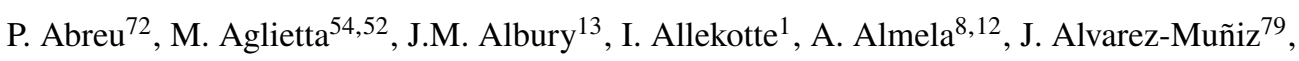
R. Alves Batista ${ }^{80}$, G.A. Anastasi ${ }^{63,52}$, L. Anchordoqui ${ }^{87}$, B. Andrada ${ }^{8}$, S. Andringa ${ }^{72}$, C. $\mathrm{Aramo}^{50}$, P.R. Araújo Ferreira ${ }^{42}$, J. C. Arteaga Velázquez ${ }^{67}$, H. Asorey $^{8}$, P. Assis ${ }^{72}$, G. Avila ${ }^{11}$, A.M. Badescu ${ }^{75}$, A. Bakalova ${ }^{32}$, A. Balaceanu ${ }^{73}$, F. Barbato ${ }^{45,46}$, R.J. Barreira Luz $^{72}$, K.H. Becker ${ }^{38}$, J.A. Bellido ${ }^{13,69}$, C. Berat ${ }^{36}$, M.E. Bertaina ${ }^{63,52}$, X. Bertou ${ }^{1}$, P.L. Biermann ${ }^{b}$, V. Binet ${ }^{6}$, K. Bismark ${ }^{39,8}$, T. Bister ${ }^{42}$, J. Biteau ${ }^{37}$, J. Blazek ${ }^{32}$, C. Bleve ${ }^{36}$, M. Boháčová ${ }^{32}$, D. Boncioli ${ }^{57,46}$, C. Bonifazi ${ }^{9,26}$, L. Bonneau Arbeletche ${ }^{21}$, N. Borodai ${ }^{70}$, A.M. Botti ${ }^{8}$, J. Brack ${ }^{d}$, T. Bretz ${ }^{42}$, P.G. Brichetto Orchera ${ }^{8}$, F.L. Briechle ${ }^{42}$, P. Buchholz ${ }^{44}$, A. Bueno ${ }^{78}$, S. Buitink ${ }^{15}$, M. Buscemi ${ }^{47}$, M. Büsken ${ }^{39,8}$, K.S. Caballero-Mora ${ }^{66}$, L. Caccianiga ${ }^{59,49}$, F. Canfora ${ }^{80,81}$, I. Caracas ${ }^{38}$, J.M. Carceller ${ }^{78}$, R. Caruso ${ }^{58,47}$, A. Castellina ${ }^{54,52}$, F. Catalani ${ }^{19}$, G. Cataldi ${ }^{48}$, L. Cazon ${ }^{72}$, M. Cerda ${ }^{10}$, J.A. Chinellato ${ }^{22}$, J. Chudoba ${ }^{32}$, L. Chytka ${ }^{33}$, R.W. Clay ${ }^{13}$, A.C. Cobos Ceruttii ${ }^{7}$, R. Colalillo ${ }^{60,50}$, A. Coleman ${ }^{93}$, M.R. Coluccia ${ }^{48}$, R. Conceição ${ }^{72}$, A. Condorelli ${ }^{45,46}$, G. Consolati ${ }^{49,55}$, F. Contreras ${ }^{11}$, F. Convenga ${ }^{56,48}$, D. Correia dos Santos $^{28}$, C.E. Covault ${ }^{85}$, S. Dasso ${ }^{5,3}$, K. Daumiller ${ }^{41}$, B.R. Dawson ${ }^{13}$, J.A. Day ${ }^{13}$, R.M. de Almeida $^{28}$, J. de Jesús ${ }^{8,41}$, S.J. de Jong ${ }^{80,81}$, G. De Mauro ${ }^{80,81}$, J.R.T. de Mello Neto ${ }^{26,27}$, I. De Mitri ${ }^{45,46}$, J. de Oliveira ${ }^{18}$, D. de Oliveira Franco ${ }^{22}$, F. de Palma ${ }^{56,48}$, V. de Souza $^{20}$, E. De Vito ${ }^{56,48}$, M. del Río ${ }^{11}$, O. Deligny ${ }^{34}$, L. Deval ${ }^{41,8}$, A. di Matteo $^{52}$, C. Dobrigkeit ${ }^{22}$, J.C. D’Olivo ${ }^{68}$, L.M. Domingues Mendes ${ }^{72}$, R.C. dos Anjos ${ }^{25}$, D. dos Santos $^{28}$, M.T. Dova ${ }^{4}$, J. Ebr ${ }^{32}$, R. Engel ${ }^{39,41}$, I. Epicoco ${ }^{56,48}$, M. Erdmann ${ }^{42}$, C.O. Escobar ${ }^{a}$, A. Etchegoyen ${ }^{8,12}$, H. Falcke ${ }^{80,82,81}$, J. Farmer ${ }^{92}$, G. Farrar ${ }^{90}$, A.C. Fauth ${ }^{22}$, N. Fazzini ${ }^{a}$, F. Feldbusch ${ }^{40}$, F. Fenu ${ }^{54,52}$,

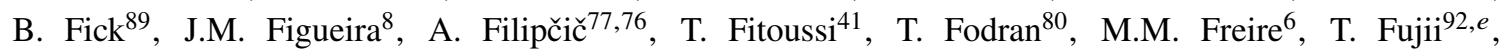
A. Fuster $^{8,12}$, C. Galea ${ }^{80}$, C. Galelli ${ }^{59,49}$, B. García ${ }^{7}$, A.L. Garcia Vegas ${ }^{42}$, H. Gemmeke ${ }^{40}$, F. Gesualdi ${ }^{8,41}$, A. Gherghel-Lascu ${ }^{73}$, P.L. Ghia ${ }^{34}$, U. Giaccari ${ }^{80}$, M. Giammarchi ${ }^{49}$, J. Glombitza ${ }^{42}$, F. Gobbi ${ }^{10}$, F. Gollan ${ }^{8}$, G. Golup ${ }^{1}$, M. Gómez Berisso ${ }^{1}$, P.F. Gómez Vitale ${ }^{11}$, J.P. Gongora ${ }^{11}$, J.M. González ${ }^{1}$, N. González ${ }^{14}$, I. Goos ${ }^{1,41}$, D. Góra ${ }^{70}$, A. Gorgi ${ }^{54,52}$, M. Gottowik ${ }^{38}$, T.D. Grubb ${ }^{13}$, F. Guarino ${ }^{60,50}$, G.P. Guedes ${ }^{23}$, E. Guido ${ }^{52,63}$, S. $\mathrm{Hahn}^{41,8}$, P. $\mathrm{Hamal}^{32}$, M.R. Hampel ${ }^{8}$, P. Hansen ${ }^{4}$, D. Harari ${ }^{1}$, V.M. Harvey ${ }^{13}$, A. Haungs ${ }^{41}$, T. Hebbeker ${ }^{42}$, D. Heck ${ }^{41}$, G.C. Hill ${ }^{13}$, C. Hojvat ${ }^{a}$, J.R. Hörandel ${ }^{80,81}$, P. Horvath ${ }^{33}$, M. Hrabovský ${ }^{33}$, T. Huege ${ }^{41,15}$, A. Insolia ${ }^{58,47}$, P.G. Isar $^{74}$, P. Janecek ${ }^{32}$, J.A. Johnsen ${ }^{86}$, J. Jurysek ${ }^{32}$, A. Kääpä ${ }^{38}$, K.H. Kampert ${ }^{38}$, N. Karastathis ${ }^{41}$, B. Keilhauer ${ }^{41}$, J. Kemp ${ }^{42}$, A. Khakurdikar ${ }^{80}$, V.V. Kizakke Covilakam $^{8,41}$, H.O. Klages ${ }^{41}$, M. Kleifges ${ }^{40}$, J. Kleinfeller ${ }^{10}$, M. Köpke ${ }^{39}$, N. Kunka ${ }^{40}$, B.L. Lago ${ }^{17}$, R.G. Lang ${ }^{20}$, N. Langner ${ }^{42}$, M.A. Leigui de Oliveira ${ }^{24}$, V. Lenok $^{41}$, A. Letessier-Selvon ${ }^{35}$, I. LhenryYvon $^{34}$, D. Lo Presti ${ }^{58,47}$, L. Lopes ${ }^{72}$, R. López ${ }^{64}$, L. Lu ${ }^{94}$, Q. Luce ${ }^{39}$, J.P. Lundquist ${ }^{76}$, A. Machado Payeras $^{22}$, G. Mancarella ${ }^{56,48}$, D. Mandat ${ }^{32}$, B.C. Manning ${ }^{13}$, J. Manshanden ${ }^{43}$, P. Mantsch ${ }^{a}$, S. Marafico ${ }^{34}$, A.G. Mariazzi ${ }^{4}$, I.C. Mariş ${ }^{14}$, G. Marsella ${ }^{61,47}$, D. Martello ${ }^{56,48}$, S. Martinelli ${ }^{41,8}$, O. Martínez Bravo ${ }^{64}$, M. Mastrodicasa ${ }^{57,46}$, H.J. Mathes ${ }^{41}$, J. Matthews ${ }^{88}$, G. Matthiae ${ }^{62,51}$, E. Mayotte ${ }^{38}$, P.O. Mazur ${ }^{a}$, G. MedinaTanco $^{68}$, D. Melo $^{8}$, A. Menshikov ${ }^{40}$, K.-D. Merenda ${ }^{86}$, S. Michal ${ }^{33}$, M.I. Micheletti ${ }^{6}$, L. Miramonti ${ }^{59,49}$, S. Mollerach ${ }^{1}$, F. Montanet ${ }^{36}$, C. Morello ${ }^{54,52}$, M. Mostafá ${ }^{91}$, A.L. Müller ${ }^{8}$, M.A. Muller ${ }^{22}$, K. Mulrey ${ }^{15}$, R. Mussa ${ }^{52}$, M. Muzio ${ }^{90}$, W.M. Namasaka ${ }^{38}$, A. Nasr-Esfahani ${ }^{38}$, L. Nellen ${ }^{68}$, M. Niculescu-Oglinzanu ${ }^{73}$, M. Niechciol ${ }^{44}$, D. Nitz ${ }^{89}$, D. Nosek ${ }^{31}$, V. Novotny ${ }^{31}$, L. Nožka ${ }^{33}$, A Nucita ${ }^{56,48}$, L.A. Núñez ${ }^{30}$, M. Palatka ${ }^{32}$, J. Pallotta ${ }^{2}$, P. Papenbreer ${ }^{38}$, G. Parente ${ }^{79}$, A. Parra ${ }^{64}$, J. Pawlowsky ${ }^{38}$, M. Pech ${ }^{32}$, F. Pedreira ${ }^{79}$, J. Pȩkala ${ }^{70}$, R. Pelayo ${ }^{65}$, J. Peña-Rodriguez ${ }^{30}$, E.E. Pereira Martins ${ }^{39,8}$, J. Perez Armand ${ }^{21}$, C. Pérez Bertolli $^{8,41}$, M. Perlin ${ }^{8,41}$, L. Perrone ${ }^{56,48}$, S. Petrera ${ }^{45,46}$, T. Pierog ${ }^{41}$, M. Pimenta ${ }^{72}$, V. Pirronello ${ }^{58,47}$, M. Platino ${ }^{8}$, B. Pont $^{80}$, M. Pothast ${ }^{81,80}$, P. Privitera ${ }^{92}$, M. Prouza ${ }^{32}$, A. Puyleart ${ }^{89}$, S. Querchfeld ${ }^{38}$, J. Rautenberg ${ }^{38}$, D. Ravignani ${ }^{8}$, M. Reininghaus ${ }^{41,8}$, J. Ridky ${ }^{32}$, F. Riehn ${ }^{72}$, M. Risse ${ }^{44}$, V. Rizi ${ }^{57,46}$, W. Rodrigues de Carvalho ${ }^{21}$, J. Rodriguez Rojo ${ }^{11}$, M.J. Roncoroni ${ }^{8}$, S. Rossoni ${ }^{43}$, M. Roth ${ }^{41}$, E. Roulet ${ }^{1}$, A.C. Rovero ${ }^{5}$, P. Ruehl ${ }^{44}$, A. Saftoiu ${ }^{73}$, F. Salamida ${ }^{57,46}$, H. Salazar ${ }^{64}$, G. Salina ${ }^{51}$, J.D. Sanabria Gomez ${ }^{30}$, F. Sánchez ${ }^{8}$, E.M. Santos ${ }^{21}$, E. Santos ${ }^{32}$, F. Sarazin ${ }^{86}$, R. Sarmento ${ }^{72}$, C. Sarmiento-Cano ${ }^{8}$, R. Sato ${ }^{11}$, 
P. Savina ${ }^{56,48,34,94}$, C.M. Schäfer ${ }^{41}$, V. Scherini ${ }^{56,48}$, H. Schieler ${ }^{41}$, M. Schimassek ${ }^{39,8}$, M. Schimp ${ }^{38}$, F. Schlüter ${ }^{41,8}$, D. Schmidt ${ }^{39}$, O. Scholten ${ }^{84,15}$, P. Schovánek ${ }^{32}$, F.G. Schröder ${ }^{93,41}$, S. Schröder ${ }^{38}$, J. Schulte ${ }^{42}$, S.J. Sciutto ${ }^{4}$, M. Scornavacche ${ }^{8,41}$, A. Segreto ${ }^{53,47}$, S. Sehgal ${ }^{38}$, R.C. Shellard ${ }^{16}$, G. Sigl ${ }^{43}$, G. Silli ${ }^{8,41}$, O. Sima ${ }^{73, f}$, R. Šmída ${ }^{92}$, P. Sommers ${ }^{91}$, J.F. Soriano ${ }^{87}$, J. Souchard ${ }^{36}$, R. Squartini ${ }^{10}$, M. Stadelmaier ${ }^{41,8}$, D. Stanca ${ }^{73}$, S. Stanič ${ }^{76}$, J. Stasielak ${ }^{70}$, P. Stassi ${ }^{36}$, A. Streich ${ }^{39,8}$, M. Suárez-Durán ${ }^{14}$, T. Sudholz ${ }^{13}$, T. Suomijärvi ${ }^{37}$, A.D. Supanitsky ${ }^{8}$, Z. Szadkowski ${ }^{71}$, A. Tapia ${ }^{29}$, C. Taricco ${ }^{63,52}$, C. Timmermans ${ }^{81,80}$, O. Tkachenko ${ }^{41}$, P. Tobiska ${ }^{32}$, C.J. Todero Peixoto ${ }^{19}$, B. Tomé ${ }^{72}$, Z. Torrès ${ }^{36}$, A. Travaini ${ }^{10}$, P. Travnicek $^{32}$, C. Trimarelli ${ }^{57,46}$, M. Tueros ${ }^{4}$, R. Ulrich ${ }^{41}$, M. Unger ${ }^{41}$, L. Vaclavek ${ }^{33}$, M. Vacula ${ }^{33}$, J.F. Valdés Galicia ${ }^{68}$, L. Valore ${ }^{60,50}$, E. Varela ${ }^{64}$, A. Vásquez-Ramírez ${ }^{30}$, D. Veberič ${ }^{41}$, C. Ventura ${ }^{27}$, I.D. Vergara Quispe ${ }^{4}$, V. Verzi ${ }^{51}$, J. Vicha ${ }^{32}$, J. Vink $^{83}$, S. Vorobiov ${ }^{76}$, H. Wahlberg ${ }^{4}$, C. Watanabe ${ }^{26}$, A.A. Watson ${ }^{c}$, M. Weber ${ }^{40}$, A. Weindl ${ }^{41}$, L. Wiencke ${ }^{86}$, H. Wilczyński ${ }^{70}$, M. Wirtz ${ }^{42}$, D. Wittkowski ${ }^{38}$, B. Wundheiler ${ }^{8}$, A. Yushkov $^{32}$, O. Zapparrata ${ }^{14}$, E. Zas ${ }^{79}$, D. Zavrtanik ${ }^{76,77}$, M. Zavrtanik ${ }^{77,76}$, L. Zehrer ${ }^{76}$

${ }^{1}$ Centro Atómico Bariloche and Instituto Balseiro (CNEA-UNCuyo-CONICET), San Carlos de Bariloche, Argentina

${ }^{2}$ Centro de Investigaciones en Láseres y Aplicaciones, CITEDEF and CONICET, Villa Martelli, Argentina

${ }^{3}$ Departamento de Física and Departamento de Ciencias de la Atmósfera y los Océanos, FCEyN, Universidad de Buenos Aires and CONICET, Buenos Aires, Argentina

${ }^{4}$ IFLP, Universidad Nacional de La Plata and CONICET, La Plata, Argentina

${ }^{5}$ Instituto de Astronomía y Física del Espacio (IAFE, CONICET-UBA), Buenos Aires, Argentina

${ }^{6}$ Instituto de Física de Rosario (IFIR) - CONICET/U.N.R. and Facultad de Ciencias Bioquímicas y Farmacéuticas U.N.R., Rosario, Argentina

${ }^{7}$ Instituto de Tecnologías en Detección y Astropartículas (CNEA, CONICET, UNSAM), and Universidad Tecnológica Nacional - Facultad Regional Mendoza (CONICET/CNEA), Mendoza, Argentina

${ }^{8}$ Instituto de Tecnologías en Detección y Astropartículas (CNEA, CONICET, UNSAM), Buenos Aires, Argentina

${ }^{9}$ International Center of Advanced Studies and Instituto de Ciencias Físicas, ECyT-UNSAM and CONICET, Campus Miguelete - San Martín, Buenos Aires, Argentina

${ }^{10}$ Observatorio Pierre Auger, Malargüe, Argentina

${ }^{11}$ Observatorio Pierre Auger and Comisión Nacional de Energía Atómica, Malargüe, Argentina

12 Universidad Tecnológica Nacional - Facultad Regional Buenos Aires, Buenos Aires, Argentina

${ }^{13}$ University of Adelaide, Adelaide, S.A., Australia

14 Université Libre de Bruxelles (ULB), Brussels, Belgium

15 Vrije Universiteit Brussels, Brussels, Belgium

${ }^{16}$ Centro Brasileiro de Pesquisas Fisicas, Rio de Janeiro, RJ, Brazil

${ }^{17}$ Centro Federal de Educação Tecnológica Celso Suckow da Fonseca, Nova Friburgo, Brazil

${ }^{18}$ Instituto Federal de Educação, Ciência e Tecnologia do Rio de Janeiro (IFRJ), Brazil

${ }^{19}$ Universidade de São Paulo, Escola de Engenharia de Lorena, Lorena, SP, Brazil

${ }^{20}$ Universidade de São Paulo, Instituto de Física de São Carlos, São Carlos, SP, Brazil

${ }^{21}$ Universidade de São Paulo, Instituto de Física, São Paulo, SP, Brazil

${ }^{22}$ Universidade Estadual de Campinas, IFGW, Campinas, SP, Brazil

${ }^{23}$ Universidade Estadual de Feira de Santana, Feira de Santana, Brazil

${ }^{24}$ Universidade Federal do ABC, Santo André, SP, Brazil

${ }^{25}$ Universidade Federal do Paraná, Setor Palotina, Palotina, Brazil

${ }^{26}$ Universidade Federal do Rio de Janeiro, Instituto de Física, Rio de Janeiro, RJ, Brazil

${ }^{27}$ Universidade Federal do Rio de Janeiro (UFRJ), Observatório do Valongo, Rio de Janeiro, RJ, Brazil

${ }^{28}$ Universidade Federal Fluminense, EEIMVR, Volta Redonda, RJ, Brazil

${ }^{29}$ Universidad de Medellín, Medellín, Colombia

${ }^{30}$ Universidad Industrial de Santander, Bucaramanga, Colombia

${ }^{31}$ Charles University, Faculty of Mathematics and Physics, Institute of Particle and Nuclear Physics, Prague, Czech Republic

32 Institute of Physics of the Czech Academy of Sciences, Prague, Czech Republic 
${ }^{33}$ Palacky University, RCPTM, Olomouc, Czech Republic

${ }^{34}$ CNRS/IN2P3, IJCLab, Université Paris-Saclay, Orsay, France

${ }^{35}$ Laboratoire de Physique Nucléaire et de Hautes Energies (LPNHE), Sorbonne Université, Université de Paris, CNRSIN2P3, Paris, France

${ }^{36}$ Univ. Grenoble Alpes, CNRS, Grenoble Institute of Engineering Univ. Grenoble Alpes, LPSC-IN2P3, 38000 Grenoble, France

${ }^{37}$ Université Paris-Saclay, CNRS/IN2P3, IJCLab, Orsay, France

38 Bergische Universität Wuppertal, Department of Physics, Wuppertal, Germany

${ }^{39}$ Karlsruhe Institute of Technology (KIT), Institute for Experimental Particle Physics, Karlsruhe, Germany

${ }^{40}$ Karlsruhe Institute of Technology (KIT), Institut für Prozessdatenverarbeitung und Elektronik, Karlsruhe, Germany

${ }^{41}$ Karlsruhe Institute of Technology (KIT), Institute for Astroparticle Physics, Karlsruhe, Germany

${ }^{42}$ RWTH Aachen University, III. Physikalisches Institut A, Aachen, Germany

${ }^{43}$ Universität Hamburg, II. Institut für Theoretische Physik, Hamburg, Germany

${ }^{44}$ Universität Siegen, Department Physik - Experimentelle Teilchenphysik, Siegen, Germany

${ }^{45}$ Gran Sasso Science Institute, L'Aquila, Italy

46 INFN Laboratori Nazionali del Gran Sasso, Assergi (L'Aquila), Italy

${ }^{47}$ INFN, Sezione di Catania, Catania, Italy

${ }^{48}$ INFN, Sezione di Lecce, Lecce, Italy

${ }^{49}$ INFN, Sezione di Milano, Milano, Italy

${ }^{50}$ INFN, Sezione di Napoli, Napoli, Italy

51 INFN, Sezione di Roma "Tor Vergata", Roma, Italy

52 INFN, Sezione di Torino, Torino, Italy

53 Istituto di Astrofisica Spaziale e Fisica Cosmica di Palermo (INAF), Palermo, Italy

54 Osservatorio Astrofisico di Torino (INAF), Torino, Italy

55 Politecnico di Milano, Dipartimento di Scienze e Tecnologie Aerospaziali , Milano, Italy

56 Università del Salento, Dipartimento di Matematica e Fisica "E. De Giorgi”, Lecce, Italy

57 Università dell'Aquila, Dipartimento di Scienze Fisiche e Chimiche, L'Aquila, Italy

58 Università di Catania, Dipartimento di Fisica e Astronomia, Catania, Italy

${ }^{59}$ Università di Milano, Dipartimento di Fisica, Milano, Italy

${ }^{60}$ Università di Napoli "Federico II", Dipartimento di Fisica "Ettore Pancini”, Napoli, Italy

${ }^{61}$ Università di Palermo, Dipartimento di Fisica e Chimica "E. Segrè", Palermo, Italy

62 Università di Roma “Tor Vergata”, Dipartimento di Fisica, Roma, Italy

${ }^{63}$ Università Torino, Dipartimento di Fisica, Torino, Italy

64 Benemérita Universidad Autónoma de Puebla, Puebla, México

${ }^{65}$ Unidad Profesional Interdisciplinaria en Ingeniería y Tecnologías Avanzadas del Instituto Politécnico Nacional (UPIITA-IPN), México, D.F., México

66 Universidad Autónoma de Chiapas, Tuxtla Gutiérrez, Chiapas, México

${ }^{67}$ Universidad Michoacana de San Nicolás de Hidalgo, Morelia, Michoacán, México

${ }^{68}$ Universidad Nacional Autónoma de México, México, D.F., México

${ }^{69}$ Universidad Nacional de San Agustin de Arequipa, Facultad de Ciencias Naturales y Formales, Arequipa, Peru

${ }^{70}$ Institute of Nuclear Physics PAN, Krakow, Poland

${ }^{71}$ University of Łódź, Faculty of High-Energy Astrophysics,Lódź, Poland

${ }^{72}$ Laboratório de Instrumentação e Física Experimental de Partículas - LIP and Instituto Superior Técnico - IST, Universidade de Lisboa - UL, Lisboa, Portugal

73 "Horia Hulubei” National Institute for Physics and Nuclear Engineering, Bucharest-Magurele, Romania

${ }^{74}$ Institute of Space Science, Bucharest-Magurele, Romania

75 University Politehnica of Bucharest, Bucharest, Romania

76 Center for Astrophysics and Cosmology (CAC), University of Nova Gorica, Nova Gorica, Slovenia

${ }^{77}$ Experimental Particle Physics Department, J. Stefan Institute, Ljubljana, Slovenia

78 Universidad de Granada and C.A.F.P.E., Granada, Spain

${ }^{79}$ Instituto Galego de Física de Altas Enerxías (IGFAE), Universidade de Santiago de Compostela, Santiago de Compostela, Spain 
${ }^{80}$ IMAPP, Radboud University Nijmegen, Nijmegen, The Netherlands

${ }^{81}$ Nationaal Instituut voor Kernfysica en Hoge Energie Fysica (NIKHEF), Science Park, Amsterdam, The Netherlands

82 Stichting Astronomisch Onderzoek in Nederland (ASTRON), Dwingeloo, The Netherlands

${ }^{83}$ Universiteit van Amsterdam, Faculty of Science, Amsterdam, The Netherlands

${ }^{84}$ University of Groningen, Kapteyn Astronomical Institute, Groningen, The Netherlands

85 Case Western Reserve University, Cleveland, OH, USA

${ }^{86}$ Colorado School of Mines, Golden, CO, USA

${ }^{87}$ Department of Physics and Astronomy, Lehman College, City University of New York, Bronx, NY, USA

${ }^{88}$ Louisiana State University, Baton Rouge, LA, USA

${ }^{89}$ Michigan Technological University, Houghton, MI, USA

${ }^{90}$ New York University, New York, NY, USA

${ }^{91}$ Pennsylvania State University, University Park, PA, USA

92 University of Chicago, Enrico Fermi Institute, Chicago, IL, USA

93 University of Delaware, Department of Physics and Astronomy, Bartol Research Institute, Newark, DE, USA

94 University of Wisconsin-Madison, Department of Physics and WIPAC, Madison, WI, USA

${ }^{a}$ Fermi National Accelerator Laboratory, Fermilab, Batavia, IL, USA

${ }^{b}$ Max-Planck-Institut für Radioastronomie, Bonn, Germany

${ }^{c}$ School of Physics and Astronomy, University of Leeds, Leeds, United Kingdom

${ }^{d}$ Colorado State University, Fort Collins, CO, USA

${ }^{e}$ now at Hakubi Center for Advanced Research and Graduate School of Science, Kyoto University, Kyoto, Japan

$f$ also at University of Bucharest, Physics Department, Bucharest, Romania 